\title{
Skin to e-skin
}

\section{Research in skin-integrated electronic technologies has the potential to produce breakthroughs in wearable health monitoring and diagnostics.}

Blurring the lines between humans and machines is the inevitable outcome of technological development and, arguably, the only chance we have of staying relevant in the world of artificial intelligence. Advances are especially relevant in the rapidly growing field of bioelectronics, resting on the idea of the seamless merging of biological systems with electronics so as to translate a variety of biological signals into electrical outputs that can be processed, analysed and acted upon. This biology-machine communication is essential to many applications such as health monitoring, diagnostics, regenerative medicine, soft robotics and wearable electronics in general.

Skin, being the largest organ of the human body and its natural barrier to the external environment, is often perceived as the ideal human-machine interface. Integrating soft sensors to the human skin may offer, for example, a minimally invasive and yet accurate way of monitoring essential biometrics including blood pressure, temperature, oxygen, blood sugar level and hydration levels. These skin-mounted devices commonly referred to as 'epidermal electronics', 'electronic tattoos' or 'electronic skin' are also suitable for applications in diagnostics and robotics owning to their ability to mimic and enhance the sensory ability of human skin.

In order to achieve these functionalities, epidermal electronics need to conform to skin topography and sustain various strains that naturally occur due to body motion. This automatically sets a number of stringent requirements for on-skin devices such as the need to be flexible, stretchable, lightweight and have long-term operational stability. Besides, water-vapour permeability and biocompatibility are very important from the user perspective as these devices should feel like a second skin, causing minimal discomfort associated with wear.

Because electronic skin has to enable a conformal contact and longterm adhesion to the epidermis with maximum flexibility and comfort, the thickness of these devices should not exceed several hundreds of nanometres. For this reason, ultrathin films made of polydimethylsiloxane, poly(vinyl alcohol), polyamide and polyethylene terephthalate are commonly used as substrates. Some latest results show devices with a total thickness less than the epidermal layer of human skin. For example, a fewmicrometre-thick photonic skin featuring polymer light-emitting diodes and organic photodetectors on an elastomeric substrate has been recently demonstrated ${ }^{1}$. Substrate-free on-skin electronics such as gold-coated nanomeshes ${ }^{2}$ made of one-dimensional polymer nanofibres enable further thickness reduction. Moreover, they are permeable to oxygen, perspiration and other bodily fluids. Although polymers are naturally flexible, their mechanical robustness is insufficient and electronic mobility too low to provide stable highperformance device operation. To tackle this issue, materials with high fracture strength ${ }^{3}$ and higher electrical conductivity have been embedded in polymeric host matrices to reinforce the composite structure by simultaneously forming a percolating conducting network. Platinum, gold, silver and various metal nanoparticles as well as semiconducting nanomaterials for example, carbon nanotubes, silicon nanomembranes, graphene $e^{4}$ and, most recently, two-dimensional transition metal dichalcogenites ${ }^{5}$ - have been used as conducting additives. Alternatively, the ability of wearable devices to accommodate various types of mechanical deformation to high levels of strain can be achieved via an 'island-bridge' approach that involves the formation of a segmented structure with rigid conducting islands electrically and mechanically connected by thin polymer bridges. This technique yields highly twistable, stretchable and bendable electronic devices characterized by long-term mechanical durability.

The implementation of these design strategies has already enabled many promising demonstrations of photonic and biochemical sensors that can be seamlessly integrated with human skin, including skin displays and a variety of electrical, chemical, pressure and temperature sensors. Besides, the field of bioelectronics has already presented a number of possible solutions for self-powering electronic skin, a highly desirable feature for wireless communication and sensing in which the human body is used as a power source by harvesting, for example, its mechanical or thermal energy.

Despite that these advances show improved skin integration, biocompatibility and

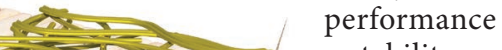
stability, current research is still far from delivering consumer-ready electronic skin. So those of us who want to lighten our pockets by having a smartphone laminated to the back of the hand or get a correct diagnosis and

timely treatment without ever seeing a doctor will have to wait a while longer. But the promise of human-machine synergy to unleash endless health benefits should be worth the wait.

It may well be that with the proliferation of health technology in full swing, the long-standing goal of living longer, healthier lives will soon become more achievable than ever. Exempt from human error and equipped with our knowledge and skill, data-driven healthcare tools may one day provide solutions for more efficient diagnostics and treatment, increasing the likelihood of successful health outcomes.

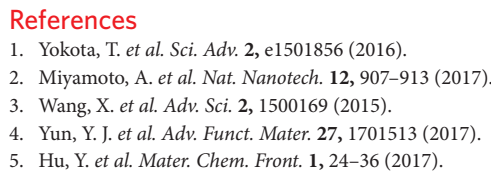

